\title{
CONVEX SOLIDS WITH PLANAR MIDSURFACES
}

\author{
VALERIU SOLTAN
}

(Communicated by Jon G. Wolfson)

\begin{abstract}
We show that the boundary of an $n$-dimensional closed convex set $B \subset \mathbb{R}^{n}$, possibly unbounded, is a convex quadric surface if and only if the middle points of every family of parallel chords of $B$ lie in a hyperplane. To prove this statement, we show that the boundary of $B$ is a convex quadric surface if and only if there is a point $p \in \operatorname{int} B$ such that all sections of $\operatorname{bd} B$ by 2 -dimensional planes through $p$ are convex quadric curves. Generalizations of these statements that involve boundedly polyhedral sets are given.
\end{abstract}

\section{INTRODUCTION AND MAIN RESULTS}

By a midsurface of a convex set in $\mathbb{R}^{n}$ we mean the locus of middle points of a family of parallel chords of the set. A classical result of convex geometry states that the boundary of a convex body $B \subset \mathbb{R}^{n}$ is an ellipsoid provided every midsurface of $B$ lies in a hyperplane (see Brunn [2, pp. 59-61] for $n=2$, Blaschke [1, p. 159] for $n=3$, and Busemann [3, p.92] for $n \geq 3$ ). Kubota [8] (for $n=2$ ) and Gruber [4] showed that the statement above remains true provided the locus of points that divide any family of parallel chords of $B$ in the same ratio lies in a hyperplane. Replacing hyperplanes by polyhedral surfaces one can obtain a joint characterization of ellipsoids and polytopes (see [12]).

It is well-known (see, e.g., [11, pp. 74-75]) that every midsurface of a quadric surface in $\mathbb{R}^{n}$, given by the equation $\sum_{i, k=1}^{n} \alpha_{i k} x_{i} x_{k}+2 \sum_{i=1}^{n} \beta_{i} x_{i}+\gamma=0$, belongs to a hyperplane. Our purpose here is to expand the characterization of ellipsoids above by considering all convex quadric surfaces.

In what follows, by convex solids in $\mathbb{R}^{n}$ we mean $n$-dimensional closed convex sets distinct from the whole space. As usual, bd $B$ and int $B$ denote, respectively, the boundary and the interior of a convex solid $B$. A convex surface in $\mathbb{R}^{n}$ is the boundary of a convex solid. This definition includes a hyperplane or a pair of parallel hyperplanes. We say that a convex surface in $\mathbb{R}^{n}$ is a convex quadric surface provided it is a part of a quadric surface. The classification of quadric surfaces (see, for example, [10]) implies that a convex quadric surface in $\mathbb{R}^{n}$ that

Received by the editors December 8, 2006.

2000 Mathematics Subject Classification. Primary 52A20.

Key words and phrases. Convex quadric surface, midsurface, planar section, polyhedron. 
contains no line can be expressed in suitable coordinates by one of the equations:

$$
\begin{array}{ll}
\alpha_{1} x_{1}^{2}+\cdots+\alpha_{n} x_{n}^{2}=1, & \text { (ellipsoid) } \\
\alpha_{1} x_{1}^{2}-\alpha_{2} x_{2}^{2}-\cdots-\alpha_{n} x_{n}^{2}=1, x_{1} \geq 0, & \text { (arm of elliptic hyperboloid on two } \\
\alpha_{1} x_{1}^{2}-\alpha_{2} x_{2}^{2}-\cdots-\alpha_{n} x_{n}^{2}=0, x_{1} \geq 0, & \text { (elliptic cone) } \\
\alpha_{1} x_{1}^{2}+\cdots+\alpha_{n-1} x_{n-1}^{2}=x_{n}, & \text { (elliptic paraboloid) }
\end{array}
$$

where all scalars $\alpha_{1}, \ldots, \alpha_{n}$ are positive. Convex quadric surfaces containing lines are both-way unbounded cylinders based on convex quadrics of the same type that lie in proper subspaces of $\mathbb{R}^{n}$. Our first result is given as follows.

Theorem 1. For a convex solid $B \subset \mathbb{R}^{n}(n \geq 2)$ distinct from a halfspace, the following conditions are equivalent:

(a) bd $B$ is a convex quadric surface,

(b) every midsurface of $B$ lies in a hyperplane,

(c) for every family $\mathcal{F}$ of parallel chords of $B$, there is a scalar $\left.\lambda_{\mathcal{F}} \in\right] 0,1[$ such that the points dividing the chords from $\mathcal{F}$ in the ratio $\lambda_{\mathcal{F}}$ lie in a hyperplane.

Analysis of the proof of Theorem 1 shows that the scalar $\lambda_{\mathcal{F}}$ in condition $(c)$ above equals $1 / 2$. Here by a chord of $B$ we mean any segment $[u, w]$ which equals the intersection of $B$ and the line $(u, w)$. The condition that $B$ be distinct from a halfspace is justified by the fact that a convex solid in $\mathbb{R}^{n}$ has chords if and only if it is not a halfspace (see [5]). Finally, we say that a segment $[u, w]$ is divided by its point $v$ in the ratio $\lambda \in[0,1]$ provided $\|u-v\|=\lambda\|u-w\|$.

Theorem 1 is a corollary of a sharper statement below, which considers families of parallel chords in a neighborhood of bd $B$. Let us recall that a line $l \subset \mathbb{R}^{n}$ is called non-recessional for $B$ if the intersection of $B$ and any translate of $l$ is either empty or a chord of $B$. For a scalar $\delta>0$ and a non-recession line $l$ of $B$ that supports $B$, denote by $B_{\delta}(l)$ the set of points in int $B$ at a distance less than $\delta$ from $l$. Let $\mathcal{F}_{\delta}(l)$ be the family of chords of $B$ each being parallel to $l$ and intersecting $B_{\delta}(l)$. A 2-dimensional convex solid $C \subset \mathbb{R}^{2}$ will be called $\delta$-polygonal provided it is locally polygonal, and for any non-recession line $l$ of $C$ that supports $C$ the set $C_{\delta}(l)$ contains at most one vertex of $C$. Obviously, any convex polygon in $\mathbb{R}^{2}$ is $\delta$-polygonal for a suitable $\delta>0$.

Theorem 2. For a convex solid $B \subset \mathbb{R}^{n}(n \geq 2)$ distinct from a halfspace, the following conditions are equivalent:

(a) either bd $B$ is a convex quadric surface or $B$ a direct sum of a subspace and a line-free closed convex set $C$ of dimension $m, 2 \leq m \leq 3$, such that $C$ is a simplicial cone if $m=3$ or $C$ is $\delta$-polygonal for a suitable $\delta>0$ if $m=2$,

(b) there is a scalar $\delta>0$ such that for any non-recession line $l$ of $B$ that supports $B$ one can find a scalar $\lambda(l) \in[0,1]$ with the following property: points that divide the chords $m \in \mathcal{F}_{\delta}(l)$ in the ratio $\lambda(l)$ lie in a hyperplane.

Analysis of the proof of Theorem 2 reveals the following remarks.

Remark 1 . The ratio $\lambda(l)$ in condition $(b)$ of Theorem 2 can take any value from $[0,1]$ if $B$ is a direct sum of a subspace and a 3-dimensional simplicial cone or a planar $\delta$-polygonal set; otherwise $\lambda(l)=1 / 2$. Thus condition $(b)$ of Theorem 2 becomes equivalent to conditions $(a)-(c)$ of Theorem 1 if we replace $[0,1]$ by $] 0,1[$. 
Remark 2. If $B$ is strictly convex (i.e., bd $B$ does not contain segments), then condition $(b)$ of Theorem 2 can be relaxed by assuming that $\delta$ may also depend on the choice of $l$. This is not true if $B$ is not strictly convex. Indeed, let $B$ be the sum of a line segment and a circle in $\mathbb{R}^{2}$. Then for any line $l$ tangent to $B$ there is a scalar $\delta=\delta(l)>0$ such that the middle points of chords $m \in \mathcal{F}_{\delta}(l)$ belong to a line.

The proof of Theorem 2 consists of two steps (see Sections 4 and 5 below): we consider the case $n=2$ separately and use Theorems 3 and 4 for the case $n \geq 3$.

Theorem 3. The boundary of a convex solid $B \subset \mathbb{R}^{n}(n \geq 3)$ is a convex quadric surface if and only if there is a point $p \in \operatorname{int} B$ such that all sections of bd $B$ by 2-dimensional planes through $p$ are convex quadric curves.

A convex solid $B \subset \mathbb{R}^{n}$ is called boundedly polyhedral (boundedly polygonal if $n=2$ ) provided its intersection with each polytope in $\mathbb{R}^{n}$ is a polytope (see [6]). The boundary of a boundedly polyhedral convex solid will be called a boundedly polyhedral surface (boundedly polygonal line if $n=2$ ).

Theorem 4. The boundary of a convex solid $B \subset \mathbb{R}^{n}(n \geq 3)$ is a convex quadric surface or a boundedly polyhedral surface if and only if there is a point $p \in \operatorname{int} B$ such that every section of bd $B$ by a 2-dimensional plane through $p$ is a convex quadric curve or a boundedly polygonal line.

We remark here that Theorem 3 is a generalization of the following statement of Busemann [3, pp. 91-92], initially proved by Kubota [7] for $n=3$ : the boundary of a convex body $B \subset \mathbb{R}^{n}(n \geq 3)$ is an ellipsoid if and only if all sections of bd $B$ by 2 -dimensional planes through a given point $p \in \operatorname{int} B$ are ellipses. Theorem 4 comprises Theorem 3 above and Theorem 4.7 from [6], which states that a convex solid $B \subset \mathbb{R}^{n}$ is boundedly polyhedral if and only if all its sections by 2 -dimensional planes through a given point $p \in \operatorname{int} B$ are boundedly polygonal.

In what follows we will be using the fact that any convex solid $B \subset \mathbb{R}^{n}$ can be uniquely expressed as the vector sum $B=M+C$, where $M$ is a subspace and $C$ is a line-free closed convex set of some dimension $m, 1 \leq m \leq n$. The affine span of a convex set $S$ (that is, the smallest plane containing $S$ ) will be denoted aff $S$ (see, e. g., [13] for general references on convex sets).

\section{Proof of Theorem 3}

Since the "if" part trivially holds, it remains to prove the "only if" part. Let $B \subset \mathbb{R}^{n}(n \geq 3)$ be a convex solid with the property that all sections of bd $B$ by 2 -dimensional planes through a given point $p \in \operatorname{int} B$ are convex quadric curves. Express $B$ as a direct sum of a subspace $M$ and a line-free closed convex set $C$ of some dimension $m$. Obviously, bd $B$ is a convex quadric surface if $1 \leq m \leq 2$. Hence we assume that $m \geq 3$. Let $q$ be the projection of $p$ on aff $C$ in the direction of $M$. Then $q$ belongs to the relative interior of $C$ and all sections of $C$ by 2dimensional planes $L \subset$ aff $C$ through $q$ are convex quadratic curves. This shows that we can reduce the proof to the case when $B$ is a line-free convex solid.

Since the case when $B$ is compact is proved in [3], we assume, in what follows, that $B$ is unbounded. Choose a hyperplane $H$ through $p$ such that the intersection $B \cap H$ is compact (this is possible because $B$ is line-free). Then [3] implies that $B \cap H$ is a solid $(n-1)$-dimensional ellipsoid. Choose in $H$ an $(n-2)$-dimensional 
plane $N$ through $p$ such that $B \cap N$ is symmetric about $p$, and let $N_{1}$ and $N_{2}$ be the $(n-2)$-dimensional planes in $H$ both parallel to $N$ and supporting $B \cap H$. Continuously rotating $H$ about $N$ (together with $N_{1}$ and $N_{2}$ ), we find a position of $H$ such that $N_{1}$ and $N_{2}$ are at the same distance from $N$. This implies that the ellipsoid $B \cap H$ is symmetric about $p$.

Let $G$ be a hyperplane parallel to $H$ and supporting $B$. Choose a point $q \in B \cap G$ and consider the halfline $l=[q, p)$. We claim that $l \subset B$. Indeed, since $B$ is unbounded, it contains a halfline $m$ with apex $q$. Let $L$ be a 2-dimensional plane containing $l \cup m$. The choice of $H$ implies that $p$ is the middle point of the chord $B \cap H \cap L$, which is parallel to the line $G \cap L$. Hence $l$ belongs to the conjugate line of $G \cap L$ with respect to the quadric curve bd $B \cap L$. Thus $l \subset B \cap L \subset B$.

Applying a suitable affine transformation, we may suppose that $q=0, l$ is perpendicular to $G$, and $B \cap H$ is an $(n-1)$-dimensional ball of radius 1 . We divide our proof into several cases.

Assume the existence of a 2-dimensional plane $L_{0}$ through $l$ such that $B \cap L_{0}$ is a convex cone. Obviously, 0 is the apex of $B \cap L_{0}$. Let $P_{1}$ and $P_{2}$ be hyperplanes that support $B$ along the sides of this cone. Consider the $(n-2)$-dimensional planes $M_{1}=G \cap P_{1}$ and $M_{2}=G \cap P_{2}$. If $L$ is a 2-dimensional plane through $l$ such that $G \cap L$ does not lie in $M_{1} \cup M_{2}$, then, as is easily seen, the section $B \cap L$ has 0 as a singular point; whence $B \cap L$ is a convex cone with apex 0 . By a continuity argument, $B \cap L^{\prime}$ is also a cone with apex 0 for any 2-dimensional plane $L^{\prime}$ through $l$ such that $G \cap L^{\prime} \subset M_{1} \cup M_{2}$. Hence $B$ is the convex cone with apex 0 generated by the $(n-1)$-dimensional ball $B \cap H$. Thus $B$ is an elliptic cone.

Now assume that no section of $B$ by a 2 -dimensional plane through $l$ is a cone. Let $K$ denote the recession cone of $B$. Then $K \subset B$ because $0 \in B$. Consider separately the following two cases: $\operatorname{dim} K \leq n-1$ and $\operatorname{dim} K=n$.

1. Let $\operatorname{dim} K \leq n-1$. Then there is a hyperplane $W$ that contains $K$. Choose a 2-dimensional plane $L$ through $l$ that does not lie in $W$. The section $B \cap L$ is a planar convex set whose recession cone belongs to the line $L \cap W$. Since bd $B \cap L$ is a convex quadric curve, it must be a parabola symmetric about $l$. By a continuity argument, the same is true for any 2-dimensional plane $L \subset W$ through $l$. If $(x, y)$ are the orthogonal coordinates in $L$ such that $l$ is the positive halfline of the $y$-axis, then $\operatorname{bd} B \cap L$ is given by an equation $y=\alpha_{L} x^{2}, \alpha_{L}>0$. We have $\alpha_{L}=\|p\|$ because bd $B \cap L$ intersects the ball $B \cap H$ with center $p$ and radius 1 at a pair of opposite points. Hence bd $B$ is an elliptic paraboloid.

2 . Let $\operatorname{dim} K=n$. Then for any 2 -dimensional plane $L$ through $l$, the recession cone of $B \cap L$ is from a halfline. Hence bd $B \cap L$ cannot be a parabola, and it cannot be a cone by the assumption above. Furthermore, since bd $B \cap L$ intersects the ball $B \cap H$ with center $p$ and radius 1 at a pair of opposite points, bd $B \cap L$ is an arm of hyperbola symmetric about $l$; whence it can be expressed in suitable orthogonal coordinates $(x, y)$ by an equation $y=\left(\alpha_{L} x^{2}+1\right)^{1 / 2}-1$. Similar to the above, $\|p\|=\left(\alpha_{L}+1\right)^{1 / 2}-1$, which implies that bd $B$ is an arm of elliptic hyperboloid on two sheets.

\section{Proof of Theorem 4}

Since the "if" part of the statement is trivial, it remains to prove the "only if" part. As in the proof of Theorem 3 we may assume that $B$ is a line-free convex solid. 
We proceed by induction on $n \geq 3$. Let $n=3$ and $B \subset \mathbb{R}^{3}$ be a line-free convex solid such that every section of bd $B$ by a 2-dimensional plane through a given point $p \in \operatorname{int} B$ is either a convex quadric curve or a boundedly polygonal line. Assume, for contradiction, that bd $B$ is neither quadratic nor polyhedral. Then there two planes through $p$, say $L$ and $M$, such that $P=\operatorname{bd} B \cap L$ is a boundedly polygonal line and $Q=\operatorname{bd} B \cap M$ is a convex quadric curve. We may assume that $B \cap L \cap M$ is a line segment, since otherwise one of $L, M$ can be replaced by a plane through $p$ whose intersection with $B$ is bounded. Because $B$ is line-free, both sections $P$ and $Q$ are line-free. We may also assume that $L$ is horizontal and $M$ is vertical. Let the part of $Q$ above $L$ and the part of $P$ in front of $M$ be bounded (see the figure below). Denote by $F$ the part of $\mathbb{R}^{3}$ situated above $L$ and in front of $M$, and let $a$ and $b$ be the endpoints of the chord $B \cap L \cap M$.

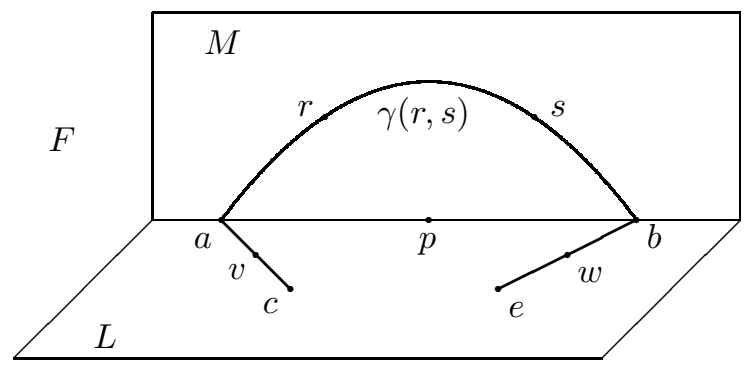

Since $P$ is locally polygonal, the $\operatorname{arc} P \cap F$ contains segments $[a, c]$ and $[b, e]$. Choose any points $v \in] a, c[$ and $w \in] b, c[$. Consider a plane $N$ through $p$ and $v$. Obviously, bd $B \cap N$ approaches $P$ when $N$ approaches $L$. Since no boundedly polygonal line can be approximated by a convex quadric curve, bd $B \cap N$ should be a boundedly polygonal line when $N$ is sufficiently close to $L$. Fix such a position of $N$ and choose a segment $\left[v, v^{\prime}\right] \subset$ bd $B \cap N$ that lies in $F$. Clearly, the plane $T$ containing both segments $[a, c]$ and $\left[v, v^{\prime}\right]$ supports $B$ and $B \cap T$ is a 2-dimensional face of $B$. From this fact it easily follows that any section of $B$ by a plane through $p$ and $v$ is boundedly polygonal. Similarly, any section of $B$ by a plane through $p$ and $w$ is boundedly polygonal.

Given a pair of points $r, s \in Q \cap F$ such that $r$ precedes $s$ clockwise along $Q$, denote by $\gamma(r, s)$ the open arc in $Q \cap F$ that joins $r$ and $s$. Let $H(r, w)$ and $H(s, v)$ denote the planes through $p, r, w$ and $p, s, v$, respectively. We are going to recursively construct two sequences of points $r_{k}, s_{k} \in \gamma(r, s), k=1,2, \ldots$, with the properties described below. Put $r_{1}=r$ and $s_{1}=s$, and assume that $r_{k}$ and $s_{k}, k \geq 1$, are chosen. Since the section bd $B \cap H\left(r_{k}, w\right)$ is boundedly polygonal, it contains a segment $\left[r_{k}, r_{k}^{\prime}\right] \subset F$. Choose a point $s_{k+1} \in \gamma\left(r_{k}, s_{k}\right)$ so close to $r_{k}$ that the plane $H\left(s_{k+1}, v\right)$ intersects the open segment $] r_{k}, r_{k}^{\prime}[$. Similarly, the section bd $B \cap H\left(s_{k+1}, v\right)$ contains a segment $\left[s_{k+1}, s_{k+1}^{\prime}\right] \subset F$. We claim that $\left[r_{k}, r_{k}^{\prime}\right]$ and $\left[s_{k+1}, s_{k+1}^{\prime}\right]$ are disjoint. Indeed, otherwise both $r_{k}$ and $s_{k+1}$ would lie in a plane that supports $B$, which is impossible because $r_{k}$ and $s_{k+1}$ belong to the boundary quadric curve $Q$. As above, there is a point $r_{k+1} \in \gamma\left(r_{k}, s_{k+1}\right)$ so close to $s_{k+1}$ that the plane $H\left(r_{k+1}, w\right)$ intersects $] s_{k+1}, s_{k+1}^{\prime}$ [ and any segment $\left[r_{k+1}, r_{k+1}^{\prime}\right] \subset \operatorname{bd} B \cap H\left(r_{k+1}, w\right) \cap F$ does not intersect $\left[s_{k+1}, s_{k+1}^{\prime}\right]$.

Furthermore, the sequences $r_{1}, r_{2}, \ldots$ and $s_{1}, s_{2}, \ldots$ can be selected such that both of them converge to a point $t \in Q$. As is easily seen, none of the curves 
$C_{1}=\mathrm{bd} B \cap H(t, v)$ and $C_{2}=\mathrm{bd} B \cap H(t, w)$ contain a segment $\left[t, t^{\prime}\right] \subset F$. The last is in contradiction with the fact that both $C_{1}$ and $C_{2}$ are boundedly polygonal. Hence the case $n=3$ is proved.

Now assume that Theorem 4 holds for all $m \leq n-1(n \geq 4)$, and let $B$ be a line-free convex solid in $\mathbb{R}^{n}$ such that every section of bd $B$ by a 2 -dimensional plane through a given point $p \in \operatorname{int} B$ is either a convex quadric curve or a boundedly polygonal line. If all these sections are convex quadric curves, then, by Theorem 3 , bd $B$ is a convex quadric surface. Assume the existence of a 2-dimensional plane $L_{0}$ through $p$ such that $B \cap L_{0}$ is boundedly polygonal, and let $L$ be any 2-dimensional plane through $p$. Choose a hyperplane $H \subset \mathbb{R}^{n}$ through $p$ that contains both $L_{0}$ and $L$. By the inductive assumption, $B \cap H$ is boundedly polyhedral; whence $B \cap L$ is boundedly polygonal. Thus all sections of $B$ by 2-dimensional planes through $p$ are boundedly polygonal, and, as proved in [6], $B$ is boundedly polyhedral.

\section{Proof of Theorem 2 For $n=2$}

$(a) \Rightarrow(b)$ If bd $B$ is a convex quadric curve, then any midcurve of $B$ lies on a line. Assume that $B$ is $\delta$-polygonal for a suitable scalar $\delta>0$. Let $l$ be a nonrecession line that supports $B$, and put $B \cap l=[u, w]$ (possibly $u=w$ ). Since $B_{\delta}(l)$ contains at most one vertex of $B$, the arc $\Gamma=\mathrm{bd} B \cap B_{\delta}(l)$ can be expressed, up to the orientation of $\mathrm{bd} B$, as the union of two or three segments: $\Gamma=] r, u[\cup] w, s[$ or $\Gamma=] r, u[\cup] w, s] \cup\left[s, t\left[\right.\right.$. In either case the left endpoints of the chords $m \in \mathcal{F}_{\delta}(l)$ belong to $] r, u[$, and we can put $\lambda(l)=0$.

$(b) \Rightarrow(a)$ We divide the proof into a sequence of lemmas, assuming that a convex solid $B \subset \mathbb{R}^{2}$ satisfies condition (b) of Theorem 2. Let us recall that $B$ is called regular provided each boundary point of $B$ belongs to a unique support line of $B$.

Lemma 1. If $B$ is not regular or is not strictly convex, then $B$ is $\delta$-polygonal.

Proof. Given a boundary point $p$ of $B$, denote by $h_{1}(p)$ and $h_{2}(p)$ the tangent halflines of $B$ with common apex $p$ such that $h_{2}(p)$ indicates the counterclockwise direction of bd $B$.

1. We first state that, given a point $p \in \mathrm{bd} B$, each of the sets $h_{1}(p) \cap B, h_{2}(p) \cap$ $B$ contains a segment, provided at least one of them does. Indeed, assume that $h_{1}(p) \cap B$ contain a segment $[q, p]$, while $h_{2}(p) \cap B=\{p\}$. By a continuity argument, there is a point $p_{0} \in \mathrm{bd} B \cap B_{\delta}(l)$ that can be reached from $p$ counterclockwise along bd $B$ such that for any point $v \in \operatorname{bd} B$ between $p$ and $p_{0}$ the following conditions hold:

1) the tangent halflines $h_{1}(p)$ and $h_{2}(v)$ are not opposite and $[p, v] \not \subset$ bd $B$,

$2)$ if $l_{v}$ is the line that contains $h_{2}(v)$, then the part of bd $B \cap B_{\delta}\left(l_{v}\right)$ that can be reached from $v$ counterclockwise is not a segment,

3) $B_{\delta}\left(l_{v}\right)$ intersects the segment $[q, p]$ along a subsegment $\left.] r, p\right]$ where $r \in[q, p[$ (this is possible because $\delta$ does not depend on $l$ ).

Given a point $v \in \operatorname{bd} B$ between $p$ and $p_{0}$, choose a scalar $\lambda\left(l_{v}\right)$ that satisfies condition (b). Conditions 1 ) and 2) above imply that $0 \neq \lambda\left(l_{v}\right) \neq 1$. Denote by $l_{v}^{\prime}$ and $l_{v}^{\prime \prime}$ the lines through $r$ and $p$, respectively, both parallel to $l_{v}$. Then left endpoints of the chords $m \in \mathcal{F}_{\delta}\left(l_{v}\right)$ that lie between $l_{v}^{\prime}$ and $l_{v}^{\prime \prime}$ belong to $[r, p]$, while the points that divide these chords in the ratio $\lambda\left(l_{v}\right)$ belong to a line given by condition $(b)$. In this case right endpoints of the chords $m \in \mathcal{F}_{\delta}\left(l_{v}\right)$ fulfill a segment $\left[r^{\prime}, p^{\prime}\right] \subset$ bd $B$. If $v$ converges to $p_{1}$, then $\left[r^{\prime}, p^{\prime}\right]$ tends to a non-trivial 
segment $[p, z] \subset$ bd $B$, which contradicts the assumption. Hence $h_{2}(p) \cap B$ contains a segment.

2. Next we state that if $p$ is a singular point of bd $B$ (i. e., if the halflines $h_{1}(p)$ and $h_{2}(p)$ are not opposite), then at least one of the sets $h_{1}(p) \cap B, h_{2}(p) \cap B$ contains a segment. Indeed, assume, for contradiction, that $h_{1}(p) \cap B=h_{2}(p) \cap B=\{p\}$. Let $l$ be the line containing $h_{2}(p)$. Choose a scalar $\lambda(l)$ that satisfies condition $(b)$. For a given chord $m=[u, w] \in \mathcal{F}_{\delta}(l)$, let $\left.v \in\right] u, w[$ satisfy $\|u-v\|=\lambda(l)\|u-w\|$. Since none of the segments $[p, u],[p, w]$ lie in $\mathrm{bd} B$, the scalar $\lambda(l)$ cannot have any of the values 0 or 1 . Hence $\lambda(l) \in] 0,1\left[\right.$. Denote by $l^{*}$ the line through $v$ and $p$. Let the points $u^{\prime}$ and $w^{\prime}$ on $l$ be such that the segments $\left[u, u^{\prime}\right],[v, p]$, and $\left[w, w^{\prime}\right]$ are parallel. Then

$$
\frac{\|u-v\|}{\|v-w\|}=\frac{\left\|u^{\prime}-p\right\|}{\left\|p-w^{\prime}\right\|}=\frac{\left\|w-w^{\prime}\right\|}{\left\|p-w^{\prime}\right\|}: \frac{\left\|u-u^{\prime}\right\|}{\left\|u^{\prime}-p\right\|} .
$$

If $[u, w]$ approaches $l$, then, by condition $(b)$, the point $v$ converges to $p$ along $l^{*}$. In this case the ratio $\left\|w-w^{\prime}\right\| /\left\|p-w^{\prime}\right\|$ tends to zero, while $\left\|u-u^{\prime}\right\| /\left\|u^{\prime}-p\right\|$ tends to a positive number by virtue of the fact that $h_{1}(p)$ and $h_{2}(p)$ are not opposite. Thus $\|u-v\| /\|v-w\| \rightarrow 0$ when $[u, w]$ approaches $l$, in contradiction with the equality $\|u-v\| /\|v-w\|=\lambda(l) /(1-\lambda(l))$. Hence at least one of the sets $h_{1}(p) \cap B$, $h_{2}(p) \cap B$ contains a segment.

The two statements above obviously imply the existence of a locally polygonal $\operatorname{arc} \Omega \subset$ bd $B$. We state that if $r, s, t$ are three consecutive vertices of $\Omega$, then $\|r-s\| \geq \delta$ and $\|s-t\| \geq \delta$. Indeed, otherwise we can find a line $l^{\prime}$ with the following properties: $(i) B \cap l^{\prime}=\{s\}$, (ii) $r, t \in B_{\delta}\left(l^{\prime}\right)$, (iii) the lines through $r$ and $t$ parallel to $l^{\prime}$ are distinct and do not contain any other vertex of $B$. Then the locus of points that divide the chords $m \in \mathcal{F}_{\delta}\left(l^{\prime}\right)$ in any given ratio $\mu$ is a polygonal line with at least three segments, which contradicts condition $(b)$. This argument also shows that $\Omega=\mathrm{bd} B$ and $B$ is $\delta$-polygonal.

If $B$ is strictly convex, then any support line of $B$ is non-recessional for $B$. In what follows, given a support line $l$ of $B$ and a scalar $\lambda(l)$ that satisfies condition $(b)$, denote by $l^{*}(\lambda)$ the line containing the locus of points that divide the chords $m \in \mathcal{F}_{\delta}(l)$ in the ratio $\lambda(l)$ and call it a conjugate line of $l$.

Lemma 2. If $B$ is regular and strictly convex, then for any tangent line $l$ of $B, a$ scalar $\lambda(l)$ that satisfies condition $(b)$ is uniquely defined and equals $1 / 2$.

Proof. First we are going to prove the uniqueness of $\lambda(l)$ and $l^{*}(\lambda)$. Indeed, assume, for contradiction, that for a certain tangent line $l$ of $B$ there are two distinct scalars $\lambda(l)$ and $\lambda^{\prime}(l)$ both satisfying condition $(b)$. The strict convexity of $B$ implies that both $\lambda(l)$ and $\lambda\left(l^{\prime}\right)$ are distinct from 0 or 1 . Let $\lambda(l)<\lambda^{\prime}(l)$. Choose a chord $[x, z] \in \mathcal{F}_{\delta}(l)$, and let the points $y, y^{\prime} \in[x, z]$ satisfy the conditions

$$
\|x-y\|=\lambda(l)\|x-z\|, \quad\left\|x-y^{\prime}\right\|=\lambda^{\prime}(l)\|x-z\| .
$$

When $[x, z]$ approaches $l$, the respective points $y$ and $y^{\prime}$ fulfill some segments on the lines $l^{*}(\lambda)$ and $l^{*}\left(\lambda^{\prime}\right)$. Since $y$ divides $\left[x, y^{\prime}\right]$ in the ratio $\lambda(l) / \lambda^{\prime}(l)$, the endpoints $z$ should lie on a line, in contradiction with strict convexity of $B$. Thus $\lambda(l)$ and $l^{*}(\lambda)$ are uniquely defined by $l$. In what follows, we write $l^{*}$ for $l^{*}(\lambda)$.

The proof of equality $\lambda(l)=1 / 2$ requires multiple steps. Choose a point $p \in$ $\mathbb{R}^{2} \backslash B$ separated by $l$ from $B$ such that $B$ has two tangent lines $l_{1}$ and $l_{2}$ through $p$ and both contact points $u_{1} \in l_{1} \cap B$ and $u_{2} \in l_{2} \cap B$ lie in $B_{\delta}(l)$. 
1. We first state that $p$ belongs to the conjugate line $l^{*}$ if and only if $\left[u_{1}, u_{2}\right]$ is parallel to $l$ and is divided by $l^{*}$ in the ratio $\lambda(l)$. Indeed, assume that $p \in l^{*}$ and choose a point $z \in \operatorname{bd} B$ such that $\left[u_{1}, z\right]$ is parallel to $l$. Then $\left[u_{1}, z\right]$ is divided by $l^{*}$ in the ratio $\lambda(l)$. Choose a chord $\left[u^{\prime}, z^{\prime}\right]$ of $B$ that is parallel to $l$ and lies between the lines $l$ and $\left(u_{1}, z\right)$. Since $\left[u^{\prime}, z^{\prime}\right]$ is divided by $l^{*}$ in the same ratio $\lambda(l)$, the lines $\left(u_{1}, u^{\prime}\right)$ and $\left(z, z^{\prime}\right)$ have a common point on $l^{*}$. Tending $\left[u^{\prime}, z^{\prime}\right]$ toward $\left[u_{1}, z\right]$ we conclude that $l_{1}$ and the tangent line of $B$ at $z$ have a common point on $l^{*}$, which is $p$. Hence $z=u_{2}$.

Conversely, let $\left[u_{1}, u_{2}\right]$ be parallel to $l$ such that $l^{*}$ intersects $\left[u_{1}, u_{2}\right]$ at a point $t$ with $\left\|u_{1}-t\right\| /\left\|u_{1}-u_{2}\right\|=\lambda(l)$. Choose any chord $\left[u_{1}^{\prime}, u_{2}^{\prime}\right]$ of $B$ that is parallel to $l$ and lies between the lines $l$ and $\left(u_{1}, u_{2}\right)$. Let $t^{\prime}$ be the point in $\left[u_{1}^{\prime}, u_{2}^{\prime}\right]$ such that $\left\|u_{1}^{\prime}-t^{\prime}\right\| /\left\|u_{1}^{\prime}-u_{2}^{\prime}\right\|=\lambda(l)$. By condition $(b)$, both $t$ and $t^{\prime}$ belong to $l^{*}$. Then the lines $\left(u_{1}, u_{1}^{\prime}\right)$ and $\left(u_{2}, u_{2}^{\prime}\right)$ have a common point on $l^{*}$. Tending $\left[u_{1}^{\prime}, u_{2}^{\prime}\right]$ toward $\left[u_{1}, u_{2}\right]$ we obtain that $l_{1}$ and $l_{2}$ have a common point on $l^{*}$, which is $p$.

2. Next we state that $\lambda\left(l_{1}\right)+\lambda\left(l_{2}\right)=1$ provided the point $p$ belongs to $l^{*}$. Indeed, let $v$ be the point at which $l$ supports $B$. Then $l^{*}=(p, v)$ as proved above. Furthermore, $l^{*}$ intersects $\left[u_{1}, u_{2}\right]$ at a point $u_{0}$ such that $\left\|u_{1}-u_{0}\right\| /\left\|u_{1}-u_{2}\right\|=\lambda(l)$. Let $\left[p_{1}, q_{1}\right]$ be a chord of $B$ parallel to $l_{1}$ and so close to $l_{1}$ that $p_{1}, q_{1} \in B_{\delta}(l)$. Choose points $p_{2}$ and $q_{2}$ in bd $B$ such that both chords $\left[p_{1}, p_{2}\right]$ and $\left[q_{1}, q_{2}\right]$ are parallel to $l$. Since both $\left[p_{1}, p_{2}\right]$ and $\left[q_{1}, q_{2}\right]$ are intersected by $l^{*}$ in the ratio $\lambda(l)$, we easily obtain that $\left(p_{2}, q_{2}\right)$ is parallel to $l_{2}$ and the lines $\left(p_{1}, q_{1}\right)$ and $\left(p_{2}, q_{2}\right)$ meet on $l^{*}$.

Denote by $r_{1}$ the point where $\left[p_{1}, q_{1}\right]$ intersects $l_{1}^{*}$, and let $r_{2}$ be the point of $\left[p_{2}, q_{2}\right]$ such that $\left[r_{1}, r_{2}\right]$ is parallel to $l$. Also, let $r_{0}$ be the point of intersection of $\left[r_{1}, r_{2}\right]$ and $l^{*}$. If the chord $\left[p_{1}, q_{1}\right]$ approaches $l_{1}$, the respective chord $\left[p_{2}, q_{2}\right]$ approaches $l_{2}$. During this movement, $r_{1}$ converges to $u_{1}$ along $l_{1}^{*}$ and $r_{0}$ converges to $u_{0}$ along $l^{*}$. This easily implies that $r_{2}$ converges to $u_{2}$ along some line $l^{\prime}$ that divides $\left[p_{2}, q_{2}\right]$ in the ratio $1-\lambda\left(l_{1}\right)$. Since the scalar $\lambda\left(l_{2}\right)$ and the respective line $l_{2}^{*}$ are uniquely determined, we have $\lambda\left(l_{2}\right)=1-\lambda\left(l_{1}\right)$ and $l^{\prime}=l_{2}^{*}$.

3. Finally, to show that $\lambda(l)=1 / 2$, consider the points $w_{1}$ and $w_{2}$ at which $l$ intersects the lines $l_{1}$ and $l_{2}$, respectively. By the argument above (with $w_{1}$ and $w_{2}$ instead of $p$ ), we have $\lambda(l)=1-\lambda\left(l_{1}\right)=1-\lambda\left(l_{2}\right)$. This and $\lambda\left(l_{1}\right)+\lambda\left(l_{2}\right)=1$ immediately imply the equality $\lambda(l)=1 / 2$.

Lemma 3. If $B$ is smooth and strictly convex, then $\mathrm{bd} B$ is a convex quadric curve.

Proof. Choose a tangent line $l$ of $B$. Keeping the notation of Lemma 2, select a point $p \in l^{*} \backslash B$ and tangent lines $l_{1}, l_{2}$ through $p$ such that the points $u_{1} \in B \cap l_{1}$ and $u_{2} \in B \cap l_{2}$ belong to $B_{\delta}(l)$. We state that the conjugate lines $l^{*}, l_{1}^{*}, l_{2}^{*}$ are either parallel or have a common point. Indeed, consider the lines $l^{*}$ and $l_{1}^{*}$. If these lines have a common point $z$, then the similarity of triangles $\Delta\left(u_{0}, u_{1}, z\right)$ and $\Delta\left(r_{0}, r_{1}, z\right)$ immediately implies the similarity of triangles $\Delta\left(u_{0}, u_{2}, z\right)$ and $\Delta\left(r_{0}, r_{2}, z\right)$. Thus $z \in l_{2}^{*}$. If the lines $l^{*}$ and $l_{1}^{*}$ are parallel, then all three segments $\left[u_{1}, r_{1}\right],\left[u_{0}, r_{0}\right]$, and $\left[u_{2}, r_{2}\right]$ are parallel, implying that $l_{2}^{*}$ is parallel to each of $l^{*}$ and $l_{1}^{*}$.

Denote by $l_{3}$ the line parallel to $\left[u_{1}, v\right]$ and supporting $B$ at a point from the $\operatorname{arc} \Gamma=\operatorname{bd} B \cap B_{\delta}(l)$. As above, the lines $l^{*}, l_{1}^{*}, l_{3}^{*}$ are either parallel or have $z$ as a common point. This argument shows the existence of a dense subset $S$ of $\Gamma$ such that the conjugate lines of tangents to $B$ at the points of $S$ are either parallel or have $z$ as a common point. By a continuity argument, this property holds for the entire arc $\Gamma$. We consider the following two cases separately. 
1. Let the conjugate lines of tangents to $B$ at $\Gamma$ be parallel. Choose in $\mathbb{R}^{2}$ a new coordinate system $(x, y)$ such that $v=0$, the $y$-axis goes along $l$ with positive direction corresponding to the clockwise orientation of $\mathrm{bd} B$, and the $x$-axis goes along $l^{*}$ with positive direction toward int $B$. Choose a point $b=(x, y) \in \Gamma \backslash 0$, and let $l_{0}$ be the line parallel to the segment $[b, 0]$ and tangent to $B$ at a point of $\Gamma$. Then the conjugate line $l_{0}^{*}$ bisects $[b, 0]$ (because $\lambda\left(l_{0}\right)=1 / 2$ ) and is parallel to $l^{*}$, i. e., to the $x$-axis. Thus the equation of $l_{0}^{*}$ is $\eta=y / 2$. Furthermore, the point $(0, y / 2)$, which is a common point of $l$ and $l_{0}^{*}$, should belong to the tangent line $l_{b}$ at $b$, given by the equation

$$
\eta-y=\frac{d y}{d x}(\xi-x)
$$

This condition results in the equation

$$
\frac{y}{2}-y=-\frac{d y}{d x} x \quad \text { or } \quad \frac{1}{x}=\frac{2}{y} \frac{d y}{d x},
$$

which gives $x=C y^{2}$. Hence $\Gamma$ is a piece of parabola.

2. Let the conjugate lines of tangents to $B$ at $\Gamma$ have a common point $z$. Since $z \in l^{*}$, we can write $z=(\alpha, 0)$ with respect to the coordinate system above. For a point $b=(x, y) \in \Gamma \backslash\{0\}$, the tangent line $l_{b}$ of $B$ at this point is given by (4.1). If $l_{0}$ is the line parallel to the segment $[b, 0]$ and tangent to $B$ at a point of $\Gamma$, then the conjugate line $l_{0}^{*}$ bisects $[b, 0]$ and contains $z$. Thus the equation of $l_{0}^{*}$ is

$$
\eta=\frac{y}{x-2 \alpha}(\xi-\alpha)
$$

whence $l_{0}^{*}$ meets the $y$-axis at $q=(0, \alpha y /(2 \alpha-x))$. As shown in Lemma $2, l_{b}$ also contains $q$. This gives

$$
\frac{\alpha y}{2 \alpha-x}-y=\frac{d y}{d x}(0-x) \quad \text { or } \quad \frac{1}{x}+\frac{1}{x-2 \alpha}=\frac{2}{y} \frac{d y}{d x} .
$$

This results in $C y^{2}=x|x-2 \alpha|$ with $C>0$. Hence $\Gamma$ is a piece of ellipse if $\alpha>0$ and a piece of hyperbola if $\alpha<0$.

Since bd $B$ can be covered by countably many pairwise overlapping arcs of the form bd $B \cap B_{\delta}(l)$, and since a piece of quadric curve is uniquely defined by a set of five points with no three on a line (see, e.g., [9, pp. 369-371]), all the pieces represent the same convex quadric curve, which is bd $B$.

\section{Proof of Theorem 2 For $n \geq 3$}

$(a) \Rightarrow(b)$ If bd $B$ is a convex quadric surface in $\mathbb{R}^{n}(n \geq 3)$, then every midsurface of $B$ lies in a hyperplane. Assume that $B$ is a direct sum of a subspace and a linefree closed convex set $C$ of dimension $m, 2 \leq m \leq 3$, such that $C$ is a simplicial cone if $m=3$ or $C$ is $\delta$-polygonal for a suitable $\delta>0$ if $m=2$. Obviously, $B$ satisfies condition $(b)$ if and only if $C$ satisfies this condition with respect to its affine span. Since the case $m=2$ is discussed in Section 4, we assume that $C$ is a 3-dimensional simplicial cone. Denote by $S_{1}, S_{2}, S_{3}$ the 2-dimensional faces of $C$. A simple argument show that for any non-recession line $l$ of $C$ there is a face, say $S_{1}$, of $C$ such that every chord of $C$ parallel to $l$ has an endpoint on $S_{1}$. Taking $\lambda(l)=0$ or $\lambda(l)=1$, we obtain that the points that divide the chords $m \in \mathcal{F}_{\delta}(l)$ in the ratio $\lambda(l)$ lie in $S_{1}$. 
$(b) \Rightarrow(a)$ Let $B \subset \mathbb{R}^{n}(n \geq 3)$ be a convex solid that satisfies condition (b) of the theorem. Choose a point $p \in \operatorname{int} B$ and a 2-dimensional plane $L$ through $p$. Select a line $l \subset L$ that is non-recessional for $B \cap L$ and supports $B \cap L$. Let $\delta>0$ and $\lambda(l) \in[0,1]$ be scalars that satisfy condition $(b)$ for $B$, and let $H$ be a hyperplane that divides the chords $m \in \mathcal{F}_{\delta}(l)$ in the ratio $\lambda(l)$. Clearly, $l=H \cap L$ is a line and each chord $m \in \mathcal{F}_{\delta}(l)$ that lies in $L$ is divided by $l$ in the ratio $\lambda(l)$. As proved in Section 4 , the relative boundary of $B \cap L$ is a convex quadric curve or a $\delta$-polygonal line.

If every section of bd $B$ by a 2-dimensional plane through $p$ is a convex quadric curve, then Theorem 3 implies that bd $B$ is a convex quadric surface. Assume that at least one such section is a $\delta$-polygonal line. Then, by Theorem $4, B$ is boundedly polyhedral; whence it can be expressed as a direct sum of a subspace $M$ and a line-free boundedly polyhedral set $C$ of some dimension $m, 1 \leq m \leq n$.

First we are going to show that $m \leq 3$. Indeed, assume for a moment that $m \geq 4$ and choose a vertex $p$ of $C$. Let $P$ be an $(m-1)$-dimensional plane in aff $C$ that separates $p$ from the other vertices of $C$ such that the section $C \cap P$ is a convex $(m-1)$-dimensional polytope that lies in the open ball $\left\{x \in \mathbb{R}^{n} \mid\|x-p\|<\delta\right\}$. Since $\operatorname{dim}(C \cap P) \geq 3$, there is a line $l^{\prime} \subset P$ with the property that for any scalar $\mu \in[0,1]$ the locus of points that divide in the ratio $\mu$ the chords of $C \cap P$ parallel to $l^{\prime}$ does not lie in an $(m-2)$-dimensional plane from $P$ (see [4]). Let $l$ be the line through $p$ and parallel to $l^{\prime}$. By the above, $l$ is a non-recession line, $C \cap P \subset B_{\delta}(l)$, and for any scalar $\mu \in[0,1]$ the locus of points that divide the chords $m \in \mathcal{F}_{\delta}(l)$ in the ratio $\mu$ does not lie in a hyperplane. Since the last contradicts condition $(b)$, we conclude that $m \leq 3$.

It remains to show that $C$ is a simplicial cone provided $m=3$. Indeed, by an argument similar to the above, any section of $C$ by a plane $L \subset$ aff $C$ close to a vertex of $C$ should be a triangle. Hence each vertex of $C$ belongs to exactly three edges of $C$. To show that $C$ is a cone, assume for a moment that $C$ has two adjacent vertices, say $u$ and $w$. Then the section of $C$ by a plane $Q$ parallel and close to $[u, w]$ is a polygon with at least four sides. Let $v$ be the middle point of $[u, w]$. An easy argument shows the existence of a support non-recession line $l$ of $B$ that contains $v$ and has direction close to that of $[u, w]$ such that $C \cap Q \subset B_{\delta}(l)$ and for any scalar $\mu \in[0,1]$ the locus of points that divide in the ratio $\mu$ the chords $m \in \mathcal{F}_{\delta}(l)$ does not lie in a hyperplane. Hence $C$ is a simplicial cone provided $m=3$.

\section{REFERENCES}

1. W. Blaschke, Kreis und Kugel, Viet, Leipzig, 1916.

2. H.Brunn, Ueber Kurven ohne Wendepunkte, Habilitationschrift, T. Ackermann, München, 1889 .

3. H. Busemann, The geometry of geodesics, Academic Press, New York, 1955. MR0075623 $(17: 779 \mathrm{a})$

4. P.M.Gruber, Über kennzeichende Eigenschaften von eucklidischen Raumen und Ellipsoiden. I, J. Reine Angew. Math. 265 (1974), 61-83. MR0338931 (49:3694)

5. V. L. Klee, Extremal structure of convex sets, Arch. Math. 8 (1957), 234-240. MR0092112 $(19: 1065 \mathrm{a})$

6. V.L.Klee, Some characterizations of convex polyhedra, Acta Math. 102 (1959), 79-107. MR0105651 (21:4390)

7. T. Kubota, Einfache Beweise eines Satzes über die konvexe geschlossene Fläche, Sci. Rep. Tôhoku Univ. 3 (1914), 235-255.

8. T. Kubota, On a characteristic property of the ellipse, Tôhoku Math. J. 9 (1916), 148-151. 
9. M. A. Penna, R. R. Patterson, Projective geometry and its applications to computer graphics, Prentice-Hall, NJ, 1986.

10. G. E. Shilov, Linear algebra, Dover Publ., New York, 1977. MR0466162 (57:6043)

11. V.Snyder, C. H. Sisam, Analytic geometry of space, Holt and Co., New York, 1937.

12. V.Soltan, Convex bodies with polyhedral midhypersurfaces, Arch. Math. 65 (1995), 336-341. MR1349188 (96h:52004)

13. R. J. Webster, Convexity, Oxford University Press, Oxford, 1994. MR1443208 (98h:52001)

Department of Mathematical Sciences, George Mason University, 4400 University Drive, Fairfax, Virginia 22030

E-mail address: vsoltan@gmu.edu 